\title{
Effect of acupuncture treatment on vascular cognitive impairment without dementia: study protocol for a randomized controlled trial
}

\author{
Bo-Feng Yang ${ }^{1,2}$, Xiang-Hong Zeng ${ }^{1}$, Yan Liu', Qing-Nan Fu' ${ }^{1}$, Tian He ${ }^{1}$, Fang Li ${ }^{1}$, Guang-Xia Shi ${ }^{1}$, Bao-Zhen Liư ${ }^{3}$,
} San-Feng Sun ${ }^{3}$, Jun Wang ${ }^{4}$, Lei Xiao ${ }^{5}$, Yan-Mei Deng ${ }^{5}$ and Cun-Zhi Liu ${ }^{1 *}$

\begin{abstract}
Background: Vascular cognitive impairment, no dementia (VCIND) is a condition at risk for future dementia and should be the target of preventive strategies. Preliminary evidence suggests that acupuncture may be a clinically effective intervention for people with early-stage vascular cognitive impairment. We will do a multicenter, 6-month, drug-controlled, nonblinded, randomized, parallel-group trial to determine whether acupuncture is effective for improving cognitive function and quality of life for patients with VCIND.

Methods/Design: A total of 216 eligible patients will be recruited and randomly assigned acupuncture for two sessions/week ( $n=108)$ or citicoline $300 \mathrm{mg} /$ day $(n=108)$ in a multicenter, 6-month trial. The primary endpoint is cognition (Alzheimer's Disease Assessment Scale, Cognitive Subscale (ADAS-cog)). Secondary endpoints include assessments of activities of daily living and behavioral symptoms (Clock Drawing Test (CDT), Activities of Daily Living $(A D L)$ and Instrumental Activities of Daily Living scale (IADL)).

Discussion: This will be the first large-scale trial specifically evaluating acupuncture therapy in VCIND. If the study confirms the effectiveness and safety of acupuncture treatment, it will be important to examine how the acupuncture approach could most effectively be integrated into the provision of routine healthcare.
\end{abstract}

Trial registration: This study is registered as an International Standard Randomised Controlled Trial on 17 January 2014, number ISRCTN 82980206.

\section{Background}

Cerebrovascular disease is the second most common cause of cognitive disorders [1]. Vascular cognitive impairment $(\mathrm{VCI})$ reflects cognitive disorders that are associated with vascular disease [2]. Some stroke-related factors such as multiple small or large strokes and ischaemic whitematter lesions have been associated with poststroke VCI. Six months after stroke, as many as 44 to $74 \%$ of patients present some degree of cognitive disturbance [3-5]. Stroke patients with cognitive impairment but no dementia have an increase in the 5-year risk of developing dementia of any type [6]. VCI with no dementia (VCIND) is a potentially treatable and preventable cause of dementia in later

\footnotetext{
* Correspondence: Icz623780@126.com

'Acupuncture and Moxibustion Department, Beijing Hospital of Traditional Chinese Medicine affiliated to Capital Medical University, 23 Meishuguanhou Street, Dongcheng District, Beijing 100010, China

Full list of author information is available at the end of the article
}

life, and familiarity with this condition will help the practitioner provide better care to patients [1]. Acupuncture is widely used for patients with cognitive disorders. It has been confirmed that acupuncture has positive effect on cognition and quality of life in patients who had a stroke [7]. However, there is no data on the effectiveness and safety of acupuncture used for VCIND patients. The aim of this study is to assess the effectiveness and safety of acupuncture in the treatment of VCIND patients.

\section{Methods/Design \\ Design}

The design is a three-center randomized trial (Figure 1). Two hundred and sixteen patients with VCIND will be recruited for inclusion in the study upon fulfillment of the selection criteria. The patients will be randomly allocated into the acupuncture treatment group (two sessions/week for 3 months) or the citicoline (Qilu Pharmaceutical Co., 


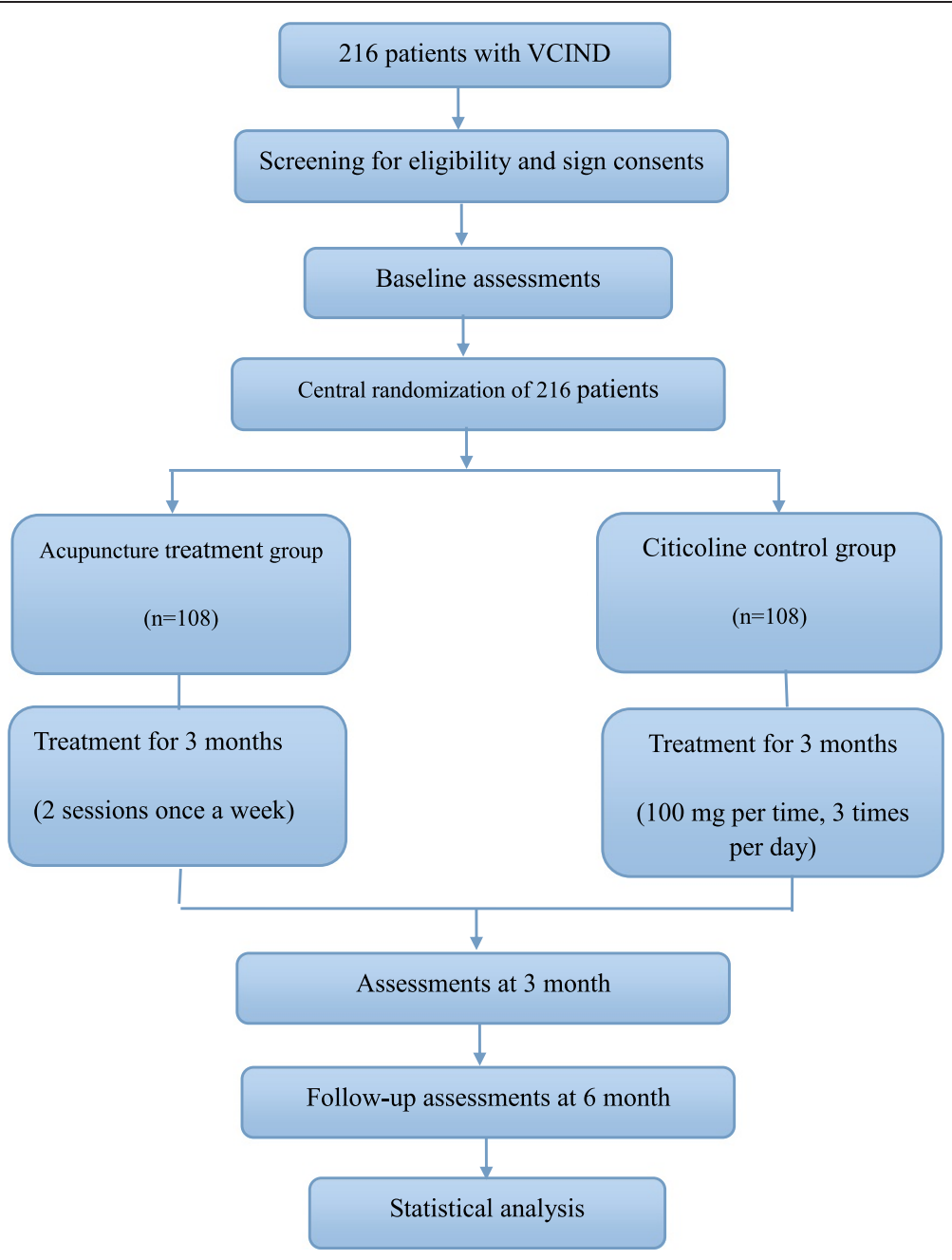

Figure 1 Flow chart.

Ltd., Shandong, China) control group (100 mg/time, three times/day for 3 months). There will be a 3-month followup period. General ethical approval has been obtained from the ethics committee of the Beijing Hospital of Traditional Chinese Medicine affiliated to Capital Medical University on 22 March, 2013 (Ref: 201317) (Additional file 1).

\section{Participants}

Patients will be recruited in acupuncture clinics in the Beijing Hospital of Traditional Chinese Medicine Affiliated to Capital Medical University, the Beijing Chinese Medicine Hospital in Huairou District, the Beijing Fengtai Hospital of Integrative Medicine and the Dongzhimen Hospital Affiliated to Beijing University of Chinese Medicine.

\section{Inclusion criteria}

Patients who meet all of the following conditions will be considered for enrollment. The inclusion criteria are as follows: subjects (males and females) age 55 to 85 years old; fluency in language sufficient to reliably complete all study assessments; a Hachinski score $\geq 7$; evidence of vascular lesions based on neuroradiology; a Mini-Mental State Examination (MMSE) score $>$ dementia threshold corrected according to the years of education (score $>17$, score $>20$ and score $>24$ for $0, \leq 6$, and $>6$ years of education, respectively); a Montreal Cognitive Assessment (MoCA) score $<25$ for $\leq 12$ years of education or score $<26$ for $>12$ years of education; and signing of an informed consent form.

\section{Exclusion criteria}

The exclusion criteria are as follows: history of mental diseases (for example, schizophrenia, serious anxiety and depression); Alzheimer's disease (AD), Parkinson's disease (PD), frontotemporal dementia (FTD) or Huntington's disease (HD); epilepsy and/or ever having antiepileptic drugs; or the presence of serious heart disease, kidney disease or liver disease. 


\section{Interventions}

After giving informed consent and completing a baseline evaluation, each participant will be randomized to the acupuncture treatment group or to the citicoline control group. The acupuncture treatment strategies are developed by consensus with experienced acupuncture practitioners and a neurologist. The acupuncture group will receive acupuncture at the Zusanli (ST36), Xuehai (SP10), Danzhong (RN17), Zhongwan (RN12), Qihai (RN6), Baihui (DU20), Fengfu (DU16), Xinshu (BL15), Yixi (BL45), Tongli (HT5) and Zhaohai (KI6) acupuncture points. Only sterile, stainless, single-use needles (Hwato, Suzhou, China) without guide tubes will be used. The gauge is $0.35 \times 40 \mathrm{~mm}$ or $0.35 \times 25 \mathrm{~mm}$ and the depth of insertion is 4 to $50 \mathrm{~mm}$, depending on the location of the needle. Needles are correctly inserted and manually stimulated until the 'De Qi' sensation is elicited. ST36, SP10, RN17, RN12, RN6, and DU16 will be inserted 10 to 15 $\mathrm{mm}$ deep with a $0.35 \mathrm{~mm} \times 40 \mathrm{~mm}$ acupuncture needle. DU20, BL15, BL45, HT5 and KI6will will be inserted 5 to $10 \mathrm{~mm}$ deep with a $0.35 \mathrm{~mm} \times 25 \mathrm{~mm}$ acupuncture needle. The needles are retained for 20 minutes in every session. Other forms of acupuncture treatment (for example, laser acupuncture, electro-acupuncture or moxibustion) will not be permitted.

The citicoline control group will receive oral citicoline $100 \mathrm{mg} /$ time, three times/day. A systematic review of the literature has revealed that citicoline is safe and effective in improving poststroke cognitive decline [8].

Treatment will be conducted over a period of 3 months, at a frequency of two sessions per week of acupuncture treatment group and three times per day for the citicoline control group.

\section{Education of acupuncturists}

The participating acupuncturists will be members who have been qualified for at least 3 years, hold a Chinese medicine practitioner license from the Ministry of Health of the People's Republic of China and take an educational course to ensure their strict adherence to the study protocol and familiarity with the administering study.

\section{Randomization}

After signed informed consent and baseline measurements have been obtained, randomization will be performed according to a random list of numbers generated with SAS software (SAS Institute, Inc., Cary, NC, USA). The allocation list will be handled by an independent investigator who has no contact with the study participants and is not involved in the supervision of staff responsible for the collection of data. The allocation of the randomization sequence will be concealed centrally by telephone.

\section{Blinding}

The interviewers who measured the outcomes will be blinded to the randomization status. It is not possible to blind participants due to the nature of the intervention. Blinding also will be maintained for data management, outcome assessment and data analysis.

\section{Primary outcome}

A general cognitive performance battery will be evaluated using the Alzheimer's Disease Assessment Scale-Cognitive (ADAS-Cog). ADAS-cog is a quantitative instrument designed to assess the severity of cognitive impairment over time in Alzheimer's disease patients, but it still is widely used for the cognitive assessment in mild to moderate vascular dementia [9]. The Chinese version of the ADAS-cog subscale has been determined to be reliable and valid among older Chinese in Hong Kong [10]. The adapted scale consists of 13 individual tests, including word recall, naming objects and fingers, commands, constructional and ideational praxis, orientation, word recognition, spoken language and comprehension, word finding and recall of test instructions. The ADAS-Cog requires approximately 30 to 45 minutes to administer, depending on the degree of cognitive impairment [11].

The ADAS-Cog will be assessed at baseline (before treatment initiation), the end of treatment and 3 months after the end of treatment.

\section{Secondary outcome measures}

Cognition of the executive function and praxis will be evaluated using the Clock Drawing Test (CDT). Executive impairments have been found to be the most common functioning impairments following stroke [12]. The CDT assesses cognition, focusing on executive function and praxis in contrast to the more language-based MiniMental State Examination (MMSE) [13].

The longitudinal functional outcome will be evaluated using the Ability of Daily Living (ADL) and Instrumental Activities of Daily Living (IADL). The functional ability of patients with vascular cognitive impairment is gradually lost [14]. Measures of functional disability typically contain items that reflect limitations in performing the ADL or IADL. Combining IADL and ADL items together in the same scale would provide enhanced range and sensitivity of measurement [15].

The CDT, ADL and IADL will be assessed at baseline (before treatment initiation), the end of treatment and 3 months after the end of treatment.

\section{Other outcomes}

The perceived credibility of treatment will be evaluated by the Treatment Credibility Scale (TCS) [16]. It is a five-item questionnaire ranging from 1 (not at all) to 5 (very confident). Items are averaged to provide a single 
treatment credibility score, with high scores reflecting high treatment credibility.

Adverse events (AEs) will be actively assessed by the trial physicians using a list at each session of acupuncture, including discomfort or bruising at the sites of needle insertion, nausea, or feeling faint after each acupuncture treatment. Adverse drug reactions like nausea and vomiting, dizziness, dry mouth, and itching will also be recorded.

The TCS and AEs will be assessed at the end of treatment.

Concomitant drugs will be recorded during the study, including those taken for the control of vascular risk factors (hypertension, diabetes, atherosclerosis, atrial fibrillation, APOE, homocysteine, etcetera), antithrombotic treatments for cardioembolism, and the use of antiplatelet agents for noncardioembolic stroke.

\section{Sample size}

According to the previous study, an improvement of ADAS-Cog scores in the treatment group and the placebo group were 1.8 (standard deviation: 5.94) and 0.3 (standard deviation: 6.32), respectively [17]. The difference in scores between the two groups was 1.5. Accordingly, we assume that a difference within 1.5 indicates acupuncture is not inferior to citicoline treatment. Using a non-inferiority design, and assuming a power of $80 \%$, an alpha value of $5 \%$, and a population standard deviation of 5.89, the required sample size is 94 patients in each group. Allowing for 15\% attrition, we should recruit 216 patients, with 108 in each group.

\section{Data analysis}

Descriptive statistics will be used to describe demographic and baseline characteristics of study participants. A generalized estimation equation (GEE) will be used to test the between-group differences. The accepted level of significance for all analyses will be $P<0.05$. Data analysis will be conducted by statisticians who are independent of the research team. Analysis will be conducted using SPSS software (SPSS 12.0 KO for Windows $\odot$ ).

\section{Discussion}

Acupuncture is a part of Traditional Chinese Medicine and has been used for thousands of years to treat clinical disorders, including stroke-related deficits. However, the possible effects of acupuncture on cognitive function have received little attention or the evidence base is poor. Acupuncture may be effective in treating some neurogenic cognitive or communicative disorders such as autism [18]. The problems associated with cognition issues after stroke continue to be challenged. This study is expected to provide evidence, with high external validity, for the effectiveness and safety of acupuncture treatment on VCIND patients. To our knowledge, this is the first large-scale, multicenter, randomized, controlled trial specifically evaluating acupuncture therapy in VCIND.

The MMSE is a brief and widely used test for screening for cognitive impairment; however, it has been criticized as a poor screening test for VCIND due to insensitivity to visuospatial and executive function impairments commonly involved in patients with cerebrovascular disease [19,20]. The Montreal Cognitive Assessment (MoCA) evaluates multiple domains of cognition, including visuo-executive, naming, attention, language, abstraction, delayed recall and orientation, but cannot completely replace the MMSE [21]. In our study, global cognitive function will be assessed using the MMSE and the MoCA in the initial screening session.

The trial has some limitations. First, in certain circumstances, it may be difficult to determine whether the cognitive decline is based on vascular factors, Alzheimer's disease, or both (mixed forms) because AD and VCI can co-occur and interact in a manner [22,23]. Second, acupuncturists and participants cannot be blinded to the group allocation, although all outcome measures will be administered and collected by an independent researcher to minimize the risk of detection bias. We will carefully discuss residual sources of bias and their potential impact on clinical outcomes when we analyze the study findings.

These results from this trial will provide preliminary evidence about the usefulness and acceptability of acupuncture for patients with VCIND, which will serve as a basis for further research.

\section{Trial status}

This trial is currently recruiting participants.

\section{Additional file}

Additional file 1: Ethical approval.

\begin{abstract}
Abbreviations
ADAS-cog: Alzheimer's Disease Assessment Scale, Cognitive Subscale; ADL: Activities of Daily Living; AEs: adverse events; CDT: Clock Drawing Test; IADL: Instrumental Activities of Daily Living Scale; MMSE: Mini-Mental State Examination; MoCA: Montreal Cognitive Assessment; TCS: Treatment Credibility Scale; VCIND: vascular cognitive impairment, no dementia.
\end{abstract}

\section{Competing interests}

The authors declare that they have no competing interests.

\section{Authors' contributions}

Conception and design, critical revision for important intellectual content and final approval of the manuscript: CZL. Conception and design, drafting the manuscript and final approval of the manuscript: BFY. Data collection and analysis and final approval of the manuscript: $X H Z, Q N F, T H, B Z L, S F S$, JW, LX, YMD. Conception and design, drafting the manuscript and final approval of the manuscript: $Y L, F L$. All authors have read and approved the manuscript. 


\section{Acknowledgements}

This trial is supported by the Beijing Municipal Science \& Technology Commission (z131107002213034).

\section{Author details}

${ }^{1}$ Acupuncture and Moxibustion Department, Beijing Hospital of Traditional Chinese Medicine affiliated to Capital Medical University, 23 Meishuguanhou Street, Dongcheng District, Beijing 100010, China. ${ }^{2}$ Tianjin University of Traditional Chinese Medicine, 312 Anshan West Road, Nankai District, Tianjin 300193, China. ${ }^{3}$ Beijing Huairou District Hospital of Traditional Chinese Medicine, 1 Houheng Street jia, Huairou District, Beijing 101400, China. ${ }^{4}$ Dongzhimen Hospital Affiliated to Beijing University of Traditional Chinese Medicine, 5 Haiyuncang, Dongcheng District, Beijing 100010, China. ${ }^{5}$ Beijing Fengtai Hospital of Integrative Medicine, 60 Changxindian Dangsanposanli jia, Fengtai District, Beijing 100072, China.

Received: 4 July 2014 Accepted: 20 October 2014 Published: 13 November 2014

\section{References}

1. O'Brien JT, Erkinjuntti T, Reisberg B, Roman G, Sawada T, Pantoni L, Bowler JV, Ballard C, DeCarli C, Gorelick PB, Rockwood K, Burns A, Gauthier S, DeKosky ST: Vascular cognitive impairment. Lancet Neurol 2003, 2:89-98.

2. Faroog MU, Gorelick PB: Vascular cognitive impairment. Curr Atheroscler Rep 2013, 15:330.

3. Madureira S, Guerreiro M, Ferro JM: Dementia and cognitive impairment three months after stroke. Eur J Neurol 2001, 6:621-627.

4. Tham W, Auchus AP, Thong M, Goh ML, Chang HM, Wong MC, Chen CP. Progression of cognitive impairment after stroke: one year results from a longitudinal study of Singaporeanstroke patients. J Neurol Sci 2002, 203-204:49-52.

5. Rasquin SM, Verhey FR, van Oostenbrugge RJ, Lousberg R, Lodder J: Demographic and CT scan features related to cognitive impairment in the first year after stroke. J Neurol Neurosurg Psychiatry 2004, 75:1562-1567.

6. Wentzel C, Rockwood K, MacKnight C, Hachinski V, Hogan DB, Feldman H, Østbye T, Wolfson C, Gauthier S, Verreault R, McDowell I: Progression of impairment in patients with vascular cognitive impairment without dementia. Neurology 2001, 57:714-716.

7. Chou P, Chu H, Lin JG: Effects of electroacupuncture treatment on impaired cognition and quality of life in Taiwanese stroke patients. J Altern Complement Med 2009, 10:1067-1073.

8. Alvarez-Sabín J, Ortega G, Jacas C, Santamarina E, Maisterra O, Ribo M, Molina C, Quintana M, Román GC: Long-term treatment with citicoline may improve poststroke vascular cognitive impairment. Cerebrovasc Dis 2013, 35:146-154.

9. Wilcock G, Möbius HJ, Stöffler A, MMM 500 group: A double-blind, placebo-controlled multicentre study of memantine in mild to moderate vascular dementia (MMM500). Int Clin Psychopharmacol 2002, 6:297-305.

10. Chu LW, Chiu KC, Hui SL, Yu GK, Tsui WJ, Lee PW: The reliability and validity of the Alzheimer's Disease Assessment Scale Cognitive Subscale (ADAS-Cog) among the elderly Chinese in Hong Kong. Ann Acad Med Singapore 2000, 4:474-485.

11. Schultz RR, Siviero MO, Bertolucci PH: The cognitive subscale of the "Alzheimer's Disease Assessment Scale" in a Brazilian sample. Braz J Med Biol Res 2001, 34:1295-1302.

12. Lamar M, Swenson R, Kaplan E, Libon DJ: Characterizing alterations in executive functioning across distinct subtypes of cortical and subcortical dementia. Clin Neuropsychol 2004, 18:22-31.

13. Peters R, Pinto EM: Predictive value of the Clock Drawing Test. A review of the literature. Dement Geriatr Cogn Disord 2008, 26:351-355.

14. Robinson DM, Keating GM: Memantine: a review of its use in Alzheimer's disease. Drugs 2006, 66:1515-1534.

15. Spector WD, Fleishman JA: Combining activities of daily living with instrumental activities of daily living to measure functional disability. J Gerontol B Psychol Sci Soc Sci 1998, 1:S46-S57.

16. Shi GX, Han LL, Liu LY, Li QQ, Liu CZ, Wang LP: Acupuncture at local and distant points for tinnitus: study protocol for a randomized controlled trial. Trials 2012, 13:224.

17. Erkinjuntti T, Kurz A, Gauthier S, Bullock R, Lilienfeld S, Damaraju CV: Efficacy of galantamine in probable vascular dementia and Alzheimer's disease combined with cerebrovascular disease: a randomized trial. Lancet 2002, 359:1283-1290.

18. Allam $H$, ElDine NG, Helmy $G$ : Scalp acupuncture effect on language development in children with autism: a pilot study. J Altern Complement Med 2008, 2:109-114.

19. Smith T, Gildeh N, Holmes C: The Montreal Cognitive Assessment: validity and utility in a memory clinic setting. Can J Psychiatry 2007, 52:329-332.

20. Nazem S, Siderowf AD, Duda JE, Have TT, Colcher A, Horn SS, Moberg PJ, Wilkinson JR, Hurtig HI, Stern MB, Weintraub D: Montreal cognitive assessment performance in patients with Parkinson's disease with "normal" global cognitionaccording to mini-mental state examination score. J Am Geriatr Soc 2009, 57:304-308.

21. Sikaroodi $H$, Yadegari S, Miri SR: Cognitive impairments in patients with cerebrovascular risk factors: a comparison of Mini Mental Status Exam and Montreal Cognitive Assessment. Clin Neurol Neurosurg 2013, 115:1276-1280.

22. Vogels RL, van der Flier WM, van Harten $B$, Gouw AA, Scheltens $P$, Schroeder-Tanka JM, Weinstein HC: Brain magnetic resonance imaging abnormalities in patients with heart failure. Eur J Heart Fail 2007, 9:1003-1009.

23. Horstmann A, Frisch S, Jentzsch RT, Müller K, Villringer A, Schroeter ML Resuscitating the heart but losing the brain: brain atrophy in the aftermath of cardiac arrest. Neurology 2010, 74:306-312.

doi:10.1186/1745-6215-15-442

Cite this article as: Yang et al:: Effect of acupuncture treatment on vascular cognitive impairment without dementia: study protocol for a randomized controlled trial. Trials 2014 15:442.

\section{Submit your next manuscript to BioMed Central and take full advantage of:}

- Convenient online submission

- Thorough peer review

- No space constraints or color figure charges

- Immediate publication on acceptance

- Inclusion in PubMed, CAS, Scopus and Google Scholar

- Research which is freely available for redistribution 